\title{
La Responsabilidad Social Corporativa como paradigma para la legitimación del orden económico neoliberal y la búsqueda de la competitividad empresarial
}

\begin{abstract}
Ramón Rueda López
Doctor en Ciencias Jurídicas y Empresariales por la Universidad de Córdoba (UCO). Docente a tiempo completo en el Departamento de Economía Aplicada, Estadística, Investigación Operativa y Organización de Empresas en la UCO. E-mail: ramon.rueda@uco.es (España).
\end{abstract}

\section{Resumen}

El debate sobre aspectos relacionados con el poder corporativo, el control del comportamiento empresarial o la legitimidad del sistema se remonta a la década de los años 50 del pasado siglo. Este debate, unido a cuál debe ser el papel que las empresas deben jugar en las sociedades contemporáneas se ha convertido en crónico en el capitalismo. Mucho más cuando en favor del crecimiento económico las empresas deben buscar permanentemente la senda de la competitividad. Tras el desarrollo teórico experimentado por la Responsabilidad Social Corporativa [RSC] durante las décadas de los 50 y 60, parece que esta se ha alzado como el paradigma más oportuno para representar, desde el final de la década de los 70 del siglo XX, la síntesis que el orden social y económico encarnado por el neoliberalismo ha encontrado para, al mismo tiempo que se dota de legitimidad al sistema a través de las empresas, alcanzar mayores niveles de competitividad empresarial.

Palabras-clave: Competitividad Responsabilidad social corporativa. Neoliberalismo. Legitimidad. 


\title{
Corporate Social Responsibility as a paradigm for the legitimation of the neoliberal economic order and the search for business competitiveness
}

\begin{abstract}
The debate on aspects related to corporate power, the control of business behavior or the legitimacy of the system goes back to the decade of the 1950s. This debate, together with what should be the role that companies should play in contemporary societies has become chronic in capitalism. Much more when in favor of economic growth companies must permanently seek the path of competitiveness. After the theoretical development experienced by the Corporate Social Responsibility [CSR] during the 50s and 60s, it seems that this has emerged as the most appropriate paradigm to represent, since the end of the 70s of the 20th century, the synthesis that the social and economic order embodied by neoliberalism has found for, at the same time that the system, through the companies, is given legitimacy, reaching higher levels of business competitiveness.
\end{abstract}

Keywords: Competitiveness. Corporate social responsibility. Neoliberalism. Legitimacy.

\section{Como referenciar em APA:}

Rueda López, R. (2019). La Responsabilidad Social Corporativa como paradigma para la legitimación del orden económico neoliberal y la búsqueda de la competitividad empresarial. R.G.Secr.,GESEC, 10(1), 141-166.

\section{Como referenciar em ABNT:}

RUEDA LÓPEZ, R. La Responsabilidad Social Corporativa como paradigma para la legitimación del orden económico neoliberal y la búsqueda de la competitividad empresarial. R.G.Secr.,GESEC, São Paulo, v. 10, n. 1, p. 141-166, jan.-abr. 2019.
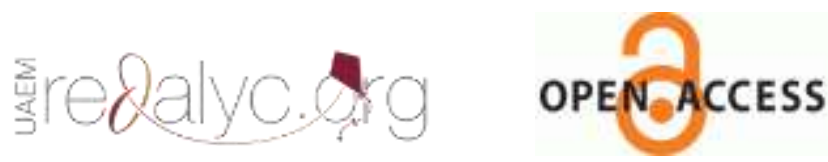


\section{Introducción}

En el final de la década de los setenta del siglo pasado, en puertas de uno de los mayores movimientos económicos mundiales que la humanidad haya conocido, la globalización neoliberal, la idea de autorregulación de las prácticas corporativas aparecía con fuerza en todas aquellas iniciativas públicas que, hasta el momento, pretendían establecer un control del comportamiento empresarial. Ejemplo de esta preocupación fue la creación, a mediados de aquella década, en el marco de la Organización de las Naciones Unidas de la Comisión de Empresas Transnacionales (Organización de las Naciones Unidas, 1974).

La interrogante que comenzaba a surgir en aquel momento era como el orden constitucional, encarnado en el Estado social y democrático de derecho, podía responder a la autorregulación, especialmente en el ámbito económico, como característica emergente en el orden jurídico. Desde la perspectiva de la propia Responsabilidad Social Empresarial ${ }^{1}$ [RSE] esta interrogante podría ser formulada en torno a la manera en la que ésta vendría a ser útil para que desde el ámbito privado, especialmente el empresarial, se asumieran y se atendieran una serie de funciones y servicios hasta ese momento reservados a los Estados.

Bajo este prisma, el Estado del bienestar comenzó a ser entendido como el conjunto de servicios que, destinados a garantizar el bienestar social, "[...] no se proporciona actual o exclusivamente por el Estado, sino por una combinación de servicios independientes, voluntarios, mutualistas y gubernamentales. En algunos casos de este tipo, el Estado o Gobierno actúa como coordinador de las provisiones y al mismo tiempo como proveedor de último recurso. En otros, el Estado puede delegar la provisión de servicios a organizaciones sociales o privadas apoyándolas financieramente [...]” (Luelmo Millán, 2012, p. 178). Este fue el modelo político y social que se ha dado en llamar la sociedad del bienestar y que, en el momento en el que el propio Estado del bienestar comenzó a ser desmantelado fue avalado por diferentes opciones política que, como sostiene Vicenç Navarro, acentuaron "la responsabilidad de la sociedad y no sólo del Estado en atender a las familias y ofrecer aquellos servicios sociales, a

\footnotetext{
${ }^{1}$ La diferencia entre RSC (responsabilidad social corporativa) y RSE (responsabilidad social empresarial) es sutil. Se utilizan indistintamente según autores (como en el presente documento de investigación) aunque cabe señalar que la RSC se refiere a todas las organizaciones independientemente de su tamaño o actividad mientras que los procesos de RSE se orientan a la actividad empresarial excluyendo otro tipo de organizaciones como son las estatáles o gubernamentales.
} 
través de la sociedad del bienestar, contraponiendo esta sociedad al Estado del bienestar" (Navarro, 2000, p. 74).

Fue en aquel contexto en el que también comenzó a surgir con fuerza el concepto de gobernanza que, a pesar de sus múltiples interpretaciones, puede ser entendido como la capacidad de conseguir que se hagan las cosas sin la competencia jurídica de mandar que sean hechas, es decir, frente a la capacidad que poseen los gobiernos a través de la autoridad de distribuir valores, la gobernanza puede hacerlo de una forma que no es autoritaria pero que es igual de efectiva (Rosenau \& Czempiel, 1992).

A través de este concepto, se trata de dar coherencia al entorno de desregulación jurídica en el que el neoliberalismo se desempeña (Santos, 2007).

Es así como frente al garantismo, "principal rasgo funcional de esa formación moderna específica que es el Estado de derecho" (Ferrajoli, 1995, p. 855), emerge con fuerza un nuevo modelo jurídico conocido como soft law o derecho blando, y que Linda Senden ha definido como: "rules of conduct that are laid down in instruments which have not been attributed legally binding force as such, but nevertheless may have certain (indirect) legal effects, and that are aimed at and may produce practical effects" (Senden, 2004, p. 112).

Así, el soft law vino a convertirse, en un momento en el que la globalización neoliberal entraba en un momento de crecimiento imparable, en el complemento perfecto de la llamada gobernanza, la cual parece exigir un Derecho más blando. Como Francisco J. Laporta San Miguel ha señalado, "gobierno es a Derecho duro, lo que gobernanza es a Derecho blando o suave" (Laporta San Miguel, 2014, p. 60).

Es en el ámbito del soft law, en el que se encuentran, como señaló Richard Reeve Baxter, aquellas clases de normas de variados grados de fuerza, de capacidad de persuadir y crear consenso que se incorporan a los acuerdos entre Estados pero no crean derechos y deberes aplicables por la fuerza. Estas normas de Derecho blando, son diferentes a las del Derecho duro consistentes en reglas de tratados que los Estados esperan que se observen y se cumplan (Baxter, 1980).

En este sentido, son las empresas las que, como sostiene Lauren Eldeman, mayores beneficios han obtenido de este Derecho blando debido, entre otras, a la presión ejercida por los lobbies empresariales y, como se defenderá más adelante, también al impulso que desde el 
ámbito de lo que se ha dado en llamar gobernanza corporativa se ha dado a normas esenciales del Derecho blando como son los códigos de conducta corporativa (Edelman, Uggen, \& Erlanger, 1999).

Así, todas aquellas declaraciones de conferencias interestatales, instrumentos de la Asamblea General de las Naciones Unidas, guías interpretativas adoptadas por organizaciones intergubernamentales, códigos de conducta, directrices y recomendaciones de organizaciones internacionales, estándares internacionales comunes adoptados por redes transnacionales de cuerpos reguladores nacionales, ONG y asociaciones profesionales e industriales, etc [...], que vienen a ser parte del Derecho blando, son las que constituyen, lo que podría denominarse, el marco normativo de la Responsabilidad Social Corporativa, y las que, a su vez, hacen que la propia RSE pase a formar parte de este entramado del soft law.

Desde este prisma, y pese a todos los buenos propósitos, lo cierto es que el marco autorregulador definido por el neoliberalismo para su nuevo orden económico mundial, es el origen de las continúas crisis financieras de carácter sistémico, constantes casos de corrupción empresarial y graves desastres ecológicos y humanos que han protagonizado la vida de la Humanidad desde entonces (Beck, 2002).

En conjunto, todos estos escándalos, desastres y crisis, generadas por la globalización neoliberal, ponen de manifiesto, no sólo una profunda crisis en el sistema capitalista sino también, la erosión de la imagen y reputación de las empresas; dos caras de una misma moneda que representaban, y representan, una poderosa fuente de inestabilidad para el orden neoliberal.

Así, mientras que para algunas personas, las más próximas a las tesis neoliberales y, sobre todo, a las neokeynesianas, esta situación tan sólo poseía un componente económico que, fruto de la perdida de confianza en el modelo, había erosionado uno de los pilares fundamentales del sistema, la competitividad (Brenner, 2009). Para otras muchas, sin embargo, el escenario señalado ponía de manifiesto una profunda crisis sistémica o de valores (Argandoña Ramíz, 2010). La diferencia entre denominaciones no es baladí.

Bajo la denominada crisis de valores el acento se pone en la falta de eticidad del propio sistema y de sus fines - la acumulación de capital — y como las consecuencias del modelo afectan a las personas y su entorno. Vivimos un tiempo, como Eduardo Galeano escribió, “en el que las buenas acciones ya no son los nobles gestos del corazón, sino las acciones que cotizan 
bien en la Bolsa, y la Bolsa es el escenario donde ocurren las crisis de valores" (Galeano, 2004, p. 248); un tiempo al que Zygmunt Bauman se refirió como el del capitalismo líquido en el que se ha desestimado la posibilidad de "evaluar — sin completo conocimiento - los medios (aquéllos disponibles y los que se consideran necesarios y deben conseguirse) para lograr el fin deseado. Se trata más bien de considerar y decidir, ante los riesgos conocidos o supuestos, cuál de los muchos fines "al alcance" (es decir, los que pueden ambicionarse razonablemente) resulta prioritario, dados los medios disponibles y tomando en cuenta sus magras posibilidades de utilidad duradera" (Bauman, 2003, p. 67).

Por otro lado, y en relación a la confianza, con Manuel Castells, se puede considerar que ésta es la "que cohesiona a una sociedad, al mercado y a las instituciones. Sin confianza, nada funciona. Sin confianza, el contrato social se disuelve y la sociedad desaparece, transformándose en individuos a la defensiva que luchan por sobrevivir" (Castells, 2012, p. 19).

Así, tras el concepto crisis de confianza, podría afirmarse que, en primer término, destaca la incapacidad del capitalismo para satisfacer las expectativas de emancipación y desarrollo social prometidas por el modernismo, algo que Jürgen Habermas, por su parte, ha denominado crisis de legitimidad (Habermas, 1999); pero, además, y quizás lo más destacado, es el rechazo y mala reputación que tanto las instituciones políticas como las organizaciones económicas y financieras cosechan entre la ciudadanía. Las primeras por haberse entregado a los intereses de la élite económica y financiera, abandonando los de la mayoría social y el consecuente empobreciendo de ésta (Castells, 2012); las segundas por, como sostiene Habermas, "tomar sus decisiones según máximas de la competencia orientada hacia la ganancia, reemplazando, así, la acción orientada por los valores por una acción regida por el interés [particular]" (Habermas, 1999, p. 51).

Con todo, la Responsabilidad Social Corporativa, más que un paradigma que pretende alcanzar una humanización de las empresas, se ha constituido como un instrumento orientado a legitimar el orden neoliberal y la búsqueda de la competitividad empresarial. Esta constituye, precisamente, la hipótesis a demostrar en esta investigación. 


\section{Metodología}

Las dos técnicas científicas empleadas en esta investigación han sido el método analítico-sintético y el análisis normativo.

El empleo del método analítico-sintético ofrece la posibilidad, en primer término, de dividir el objeto de estudio en los principales elementos que lo componen para, a partir de los mismos, abordar su análisis y poder determinar sus características y particularidades (Bunge, 1980).

De esta manera será posible abordar dos trayectorias investigadoras. La primera que permitirá presentar los principales hitos políticos, sociales y económicos a través de los cuales se ha pretendido la reconstrucción de la confianza sistémica a través de la reputación corporativa. La segunda presentará, a su vez, el paradigma de la RSE desde su génesis hasta su conversión en una herramienta al servicio de la competitividad empresarial.

Por su parte, la síntesis permite integrar, cada una de estas particularidades y características, desde una perspectiva integral e interrelacionada lo que lleva a comprender el objeto de estudio en su dimensión plena y conjunta.

En cuanto al método de análisis normativo empleado, su aplicación viene justificada por la necesidad de determinar las dinámicas jurídicas que, desde el ámbito internacional y nacional, han permitido dictar las normas que han concretado los valores y principios éticos y que, a su vez, han hecho posible el funcionamiento del orden social y político hegemónico en cada momento, particularmente en el ámbito de la educación (Carnelutti, 1940).

Las fuentes documentales — primarias y secundarias - consultadas, especialmente las bibliográficas, son numerosas, lo que ha dado lugar a una importante revisión de las mismas. A pesar de no ser esta una investigación positivista, no se ha eludido la consulta de doctrina, normativa y jurisprudencia. Se ha cuidado plantear la argumentación sociológica, política y filosófica a la vez que presentar los marcos normativos de referencia en cada uno de los aspectos abordados. 


\section{Marco teórico}

3.1 La reconstrucción de la confianza en el sistema y la reputación corporativa: el autocontrol corporativo

Desde que en la década de 1950 del siglo pasado comenzase un interesante e incesante debate, sobre todo académico, en relación a un problema de doble cara: como legitimar el poder que las empresas ya atesoraban en aquel momento y cual debía ser el papel que las mismas debían desempeñar en la sociedad (McGuire, 1963), las propuestas surgidas en los ámbitos social, político y económico para dar respuesta a estas cuestiones no han cesado.

Sin embargo no fue hasta 1992, cuando, en su caso, las autoridades financieras del Reino Unido, después de varios escándalos de corrupción financiera que erosionaron la credibilidad y confianza de la sociedad británica en sus empresas, publicaron un informe titulado The Financial Aspects of Corporate Governance, llamado coloquialmente, Cadbury Report, o Informe Cadbury (Dempsey, 2013).

La intención de este informe era clara: resultaba necesario que las empresas británicas asumieran nuevos estándares de gestión empresarial con el propósito de recuperar la confianza de las personas inversoras, facilitando así el desarrollo de sus negocios y fortaleciendo, por tanto, la posición competitiva de la economía británica (Committee on the financial aspects of corporate governance, 1992).

El Informe Cadbury introdujo el concepto de gobernanza corporativa o gobierno corporativo como el sistema por el cual las empresa son dirigidas y controladas. Desde esta perspectiva el objetivo del Informe Cadbury era muy concreto: ofrecer a las empresas recomendaciones y pautas de gobierno corporativo que, más allá de las obligaciones que la legislación dicta en cuanto a las funciones y tareas que los órganos de dirección y gestión empresarial (juntas de accionistas y consejo de administración) deben cumplir y respetar, permitiese, a las mismas, alcanzar altos niveles de comportamiento corporativo (Committee on the financial aspects of corporate governance, 1992).

Mediante un conjunto de recomendaciones y pautas de carácter económico y financiero, el Informe vino a introducir en aquel momento los Códigos de Buenas Prácticas. Códigos que, como bien deja indicado el propio informe, poseen un carácter voluntario, en ningún caso las 
empresas están obligadas a su cumplimiento. Eso si, el Informe sostiene, tajantemente, que es preferible un sistema voluntario como el propuesto a la capacidad legislativa que los gobiernos pueden ejercer frente a las malas prácticas corporativas (Committee on the financial aspects of corporate governance, 1992).

Pero mucho más que este carácter voluntario, lo que realmente destaca en el Informe Cadbury es que introduce, por primera vez en el ámbito del soft law, un criterio por el cual una vez que las empresas aceptan las recomendaciones y compromisos recogidos en el Código de Buenas Prácticas pueden no cumplir con dicho acuerdo, explicando, en este caso, las motivación que llevan a dicho incumplimiento (Committee on the financial aspects of corporate governance, 1992). Este es el que se ha dado en conocer como principio de cumplir o explicar.

Un principio que, por otro lado, ha quedado consagrado en el acervo comunitario a través del artículo 20.1 b) de la Directiva 2013/34/UE, donde se establece que "cuando una empresa, de conformidad con el Derecho nacional, no aplique el código de gobernanza empresarial [...] realizará una indicación de qué partes del código de gobernanza empresarial no aplica y las razones para ello; cuando la empresa haya decidido no atenerse a alguna disposición del código de gobernanza empresarial [...], explicará las razones de su actuación”. (Unión Europea, 2013: 39).

Así, la publicación del Informe Cadbury trajo consigo la consagración de un modelo jurídico híbrido en el que la gobernanza corporativa se fundamenta, primero, en el marco jurídico y legislativo aprobado por cada gobierno y, segundo, por los códigos de conducta voluntarios (Dempsey, 2013).

Por otro lado es necesario señalar que la crisis de legitimidad del capitalismo tenía abierto un poderoso frente. Desde los informes que en 1970 comenzaron a publicar entidades como el Counter Information Services [CIS], hasta las investigaciones como el "Informe Lugano" (George, 2002) o "No Logo: el poder de las marcas" (Klein, 2001) fueron muchas y diversas las investigaciones que pusieron de manifiesto los peligros que para el planeta y las personas entrañaban la globalización neoliberal y determinadas prácticas empresariales.

La presión ejercida por la sociedad civil organizada en torno a ONG, sindicatos, algunos grupos políticos, grupos de presión, artistas, personas investigadoras, [...] hizo que las grandes entidades internacionales y las grandes corporaciones asumieran la necesidad de que algo 
debían cambiar en su comportamiento (Cortina Orts, 2003; Cuesta González, 2004; Klein, 2001; Satorras Fioretti, 2008).

Parecía, así, que a la burguesía internacional, como ya sucediera en el siglo XIX, le había salido un duro competidor: el movimiento anti-globalización o alter-globalización, como también se le conoce. Este movimiento parecía capaz, en aquel contexto, de hacer sombra a la hegemonía neoliberal, de deslegitimar y cuestionar el poder de las empresas transnacionales. No en vano, los entonces ultraliberales gobiernos de Latinoamérica, donde nació la Primavera Social de Porto Alegre (Seoane \& Taddei, 2001), acabaron sucumbiendo y perdiendo el poder frente a la presión que, especialmente durante la década de 1990, ejercieron diversos movimientos sociales y fuerzas políticas progresistas (Veltmeyer \& Petras, 1998).

Desde otro ángulo, la ONU apostó, especialmente durante la década de los noventa del pasado siglo, por superar la tensión entre los intereses de las empresas transnacionales y los de la mayoría social del planeta. Este esfuerzo dio lugar a que, en la órbita de la ONU, proliferasen los llamamientos, resoluciones, declaraciones y acuerdos internacionales - muchos de ellos analizados en el capítulo primero- en los que se pedía, particularmente a las empresas, la eliminación del despido, la reducción de la pobreza, la creación de la jornada intensiva, la promoción de la igualdad de oportunidades, el refuerzo del papel de la mujer, el establecimiento de mejoras en la libertad humana y la integración social y la protección de la infancia (Gladwin, Krause, \& Kennelly, 1995).

El resultado final de todas estas iniciativas fue el llamamiento que el Secretario General de la ONU, Kofi Annan, hizo durante su participación en la novena Asamblea Anual del Foro Económico Mundial de 1999 para la creación de lo que denominó UN-Global Compact (o Pacto Mundial como es conocido en España). El propósito de esta propuesta no era otro que el de crear una alianza mundial con las empresas transnacionales para compartir valores y principios de carácter ético en las áreas de los derechos humanos, las normas laborales, las prácticas ambientales y la corrupción, que dotaran al capitalismo global de un rostro más humano (Annan, 1999). Esta propuesta, finalmente, sería presentada y aprobada en marzo del año 2000 en el marco de la Asamblea General de las Naciones Unidas (Organización de las Naciones Unidas, 2005). 
El UN-Global Compact nacía con el respaldo de diversas agencias multilaterales de la ONU, configurándose como una red de trabajo descentralizada en cuya gestión y decisiones operativas confluyen actores públicos y privados. Esto ha permitido, según señala Rosa $\mathrm{M}^{\mathrm{a}}$ Satorras Fioretti, avanzar en la creación de una ciudadanía corporativa global que aspira a superar las tensiones entre las expectativas de las ETN, la sociedad civil y la propia comunidad internacional (Satorras Fioretti, 2008).

Pero también y no menos relevante, fue la aprobación en el año 2003 de la Convención de la ONU contra la Corrupción. Con esta Convención la ONU mostraba su preocupación "por la gravedad de los problemas y las amenazas que plantea la corrupción para la estabilidad y seguridad de las sociedades al socavar las instituciones y los valores de la democracia, la ética y la justicia y al comprometer el desarrollo sostenible y el imperio de la ley" (Organización de las Naciones Unidas, 2003, p. 2), proponiendo un conjunto de normas y reglamentos que, como señaló Annan en una edición de la Convención publicada en el año 2004, “pueden aplicar todos los países para reforzar sus regímenes jurídicos y reglamentarios destinados a la lucha contra la corrupción” (Organización de las Naciones Unidas, 2004, Prefacio).

Con todo, lo cierto es que, de manera global, durante la década de los 80 y los 90, se despertó una nueva conciencia ética que comenzó a demandar un cambio de paradigma en cuanto al papel y la responsabilidad de las grandes corporaciones (Sanahuja Perales, 2014; Waddock, 2008) y que, en absoluto, se correspondía con las propuestas recogidas, por ejemplo, en el marco del Informe Cadbury.

Desde aquel momento, la RSE comenzó a formar parte, no sólo de la praxis empresarial, sino también de los discursos y las propuestas políticas. La RSE, además, como consecuencia de sonados escándalos financieros y de prácticas corruptas, comenzó a ser percibida como un importante vector de transmisión de la llamada ética empresarial. El capitalismo y sus más importantes agentes, las empresas, precisaban no sólo de un lavado de cara, sino de legitimar su sentido y su praxis, a esto sería convocada la RSE.

3.2 Génesis y evolución del paradigma de la Responsabilidad Social Corporativa en el orden neoliberal

Como se ha expuesto, la crisis de legitimación del capitalismo y, al mismo tiempo, de reputación de las empresas ha sido una constante durante décadas. Esta es la idea que sirvió a 
Howard R. Bowen para comenzar a reclamar en 1953 la necesidad de construir un nuevo marco relacional que, sobre un fundamento ético, pudiera armonizar los fines de las empresas y los de la sociedad.

En este sentido, en su libro Social Responsibilitties of Businessmen — con el que se marca el inicio del desarrollo del concepto contemporáneo de Responsabilidad Social Corporativa-, consideró que, dado que los problemas éticos con frecuencia surgen cuando los intereses individuales y sociales están en conflicto, resulta del todo obligatorio que la ingeniería económica asuma la necesidad de buscar las sinergias entre el interés individual y el interés social de tal modo que, ambos, sean compatibles entre si.

Pero Bowen, además de analizar la relación entre las empresas y la sociedad, ofreció la que podría ser considerada como la primera definición de Responsabilidad Social Corporativa, entendiendo ésta como las obligaciones y decisiones que los hombres de negocios deben asumir, en relación a la política de empresa, para seguir las líneas de acciones que son deseables en términos de los objetivos y valores de la sociedad y de su entorno (Bowen, 1953).

En términos similares, pero más actuales y concretos en relación al ámbito de la empresa, se expresan Ildefonso Camacho Laraña y Lina Gálvez Muñoz.

Para Camacho Laraña la empresa “es una institución económica y, por consiguiente, no puede excluirse de sus objetivos la búsqueda de un rendimiento económico: eso es precisamente lo que la distingue de cualquier otro tipo de organización. Pero este rendimiento no puede obtenerse a cualquier precio: no puede obtenerse al precio de un perjuicio notable para la sociedad, como sería, [entre otras], la reducción del ciudadano a sumiso consumidor o de sus trabajadores a puros instrumentos de producción” (Camacho Laraña, Fernández Fernández, \& Miralles Massanés, 2009, pp. 27-28).

Por otra parte, Gálvez Muñoz señala que “es necesario considerar la empresa como algo más que una simple función de producción para poder incorporar a otros, [como las mujeres], en su análisis” (Gálvez Muñoz, 2000, p. 147). Mediante esta idea, Gálvez Muñoz no sólo quiere poner de manifiesto la necesidad de ampliar el objeto de estudio en el mundo de las empresas, sino que además, incorporando a las mujeres, será posible comenzar a definir una nueva naturaleza empresarial y, por tanto, el nuevo papel que las empresas deben desempeñar en la sociedad (Gálvez Muñoz, 2004). 
Se trataría, por tanto, de dirigir la práctica empresarial hacia una nueva senda en la que un nuevo consenso o alianza social, fundado sobre lo que Beck ha llamado un sistema normativo de reglas de responsabilidad social, indemnización y precauciones, capaz de crear seguridad en el momento presente frente a un futuro incierto y abierto y de, en definitiva, sustituir al egoísmo ético, dominante desde las tesis económicas diseñadas por Adam Smith hace tres siglos (Beck, 2002).

Alcanzar esta alianza es lo que, de alguna forma, han perseguido las personas que han abogado por el paradigma de la Responsabilidad Social Corporativa desde el instante en el que ésta comenzó a ser conceptualizada por Bowen. Al menos este ha sido el objetivo hasta que, como se revelará más adelante, el neoliberalismo se alzase como corriente de pensamiento dominante.

Durante la década de los sesenta del pasado siglo, el desarrollo de la RSE fue enriquecido con las aportaciones de investigadores como Joseph W. McGuire y Keith Davis entre otros. Ambos comenzaron a entender la Responsabilidad Social Corporativa como un nuevo paradigma en la gestión empresarial, útil, especialmente, para legitimar el poder que las empresas ejercían, y aún ejercen, en en la sociedad (Aras y Crowther, 2010).

Esta fue la conclusión a la que llegó McGuire, quien después de analizar las relaciones empresariales en la sociedad en términos de relaciones de poder, legitimidad y burocratización, consideró que: "the idea of social responsibilities supposes that the corporation has not only economic and legal obligations, but also certain responsibilities to society which extend beyond these obligations. The corporation today must take an interest in politics, in the welfare of the community, in education, in the happiness of its employees - in fact, in the whole social world about it. In a sense, therefore, it must act "justly" as a proper citizen should" (McGuire, 1963: 144).

Por su parte Davis — uno de los investigadores más prolijos y cuyas aportaciones, aún hoy en día, siguen influyendo en el desarrollo teórico de la Responsabilidad Social Corporativa (Carroll, 2008) - y Robert Blomstrom, definieron la RSC como "a person's obligation to consider the effects of his decisions and actions on the whole social system. Businessmen apply social responsibility when they consider the needs and interest of others who may be affected 
by business actions. In so doing, they look beyond their firm's narrow economic and technical interests" (Davis \& Blomstrom, 1966, p. 12).

Esta definición, al reconocer de facto la influencia y el poder que las empresas ejercen en la sociedad, les llevó a considerar lo que ambos llamaron la ley de hierro de la responsabilidad social: "in the long run, those who do not use power in a manner which society considers responsible will tend to lose it" (Davis \& Blomstrom, 1966, p. 174-175).

Esta vez en solitario, Davis publicó en 1967 un artículo titulado "Understanding the Social responsibility puzzle: what does the businessman owe to society?" Con este artículo señaló que cualquier organización, ya sea empresarial o no, debe asumir su responsabilidad social, definiendo, a continuación, los siguientes elementos como fundamentales en el concepto de Responsabilidad Social Corporativa: “[...] business is a joint venture of responsible citizens and groups of citizens, such as investors, managers, workers, communities, scientists, and others. Together these groups offer diverse inputs and expect diverse outputs" (Davis, 1967, p. 47), dando, así, un nuevo impulso al desarrollo de las ideas en torno a los grupos de interés.

La RSE es una herramienta útil para equilibrar el poder que las empresas poseen en la sociedad, lo que en definitiva supone la legitimación y fortalecimiento de la su imagen corporativa en relación a los y las consumidoras, así como su posición competitiva en el mercado; esto implicó un cambio sustancial en la concepción de la responsabilidad social, ya que si hasta ese momento ésta había sido considerada como una acción individual de los hombres de negocios, a partir de ese momento pasaba a ser considerada como parte de la política de empresa (Davis, 1967).

La autorregulación es la fórmula que, fundamentada en la experiencia y el compromiso social puesto de manifiesto por las personas que dirigen la empresa, debían adoptar las empresas para la gestión e implementación de las prácticas de responsabilidad social (Davis, 1967).

Por otra parte, Davis fue, quizás, el que mejor enmarcó, hasta ese momento, el componente ético de la Responsabilidad Social Corporativa. Así, señalando que la esencia de la RSE nace de la preocupación corporativa por las consecuencias éticas que sus actos tienen en cualquier parte la sociedad, Davis, no sólo dotó de sentido ético a la RSE sino que, además, la vinculaba directamente con la ética de la responsabilidad que Weber había propuesto décadas antes (Davis, 1967). 
Sin embargo, aquellos fueron los años en los que, nuevamente, se reavivó el enfrentamiento académico entre quienes, como Levitt o Friedman, pensaban que la RSE era un factor que alejaba a la empresa de su objetivo — así se volvió a manifestar Friedman en 1970 en su archiconocido artículo "The social responsibility of Business is to increase its profits" (Friedman, 1970) - , y entre quienes consideraban que la empresa, cuya actividad se desarrolla en una economía cada vez más globalizada y cambiante, debe asumir un comportamiento más responsable en relación a su entorno.

Pero en contra de lo planteado por Friedman las personas que apostaban por su evolución no cejaban en su empeño. Este fue el caso de Raymond A. Bauer y Dan H. Fenn, quienes dieron un impulso decidido a otro de los elementos claves de la Responsabilidad Social Corporativa: la auditoria o balance social.

Con la publicación de su artículo "What is the corporate social audit?", estos investigadores consideraron que la sociedad, a fin de reforzar su creencia en la necesidad de que las empresas impulsen su responsabilidad social, debe exigir algún tipo de contabilidad o información de la actuación de las empresas en áreas no económicas (Bauer \& Fenn, 1973). Pero además, y quizás lo más importante, ofrecieron una definición que, heredera de las tesis de Bowen, consideró a la auditoria social como: " a commitment to systematic assessment of and reporting on some meaningful, definable domain of a company's activities that have social impact” (Bauer \& Fenn, 1973, p. 38).

Con todo, desde los primeros postulados de Bowen hasta casi los años ochenta el debate a cerca de la RSE había estado marcado por una orientación marcadamente teórica. Este debate, bien reflejado en el artículo de Davis titulado "The case for and against business assumption of social responsibilities", quedó polarizado, principalmente, en torno a dos argumentos ya clásicos: el de la maximización del beneficio a corto plazo, en aquellas posturas en contra y el del beneficio a largo plazo y sostenible para aquellas otras posiciones favorables (Davis, 1973).

Sin embargo, como el mismo Davis recogió en este artículo, la RSE, ya en aquel momento, se había convertido en el sello distintivo de una civilización global madura. Señalando, al mismo tiempo, que aquellas empresas que no integraran en su gestión y decisiones sus compromisos sociales acabarían por engrosar los ranking de aquellas empresas con mala imagen o reputación (Davis, 1973). 
Como se ha mencionado, en la década de los setenta del siglo XX, parecía que la RSE estaba llamada a ser el paradigma que dotase de legitimidad y buena reputación al capitalismo y sus principales agentes económicos: las empresas.

En el debate sobre la RSE de aquella década quedaría cerrada con las aportaciones realizadas por Carroll en 1979. En el artículo que publicó aquel año propuso un modelo denominado Social Performance Model. A través de este modelo no sólo pretendía explicar las motivaciones, obligaciones y aspectos sociales que han de guiar la actuación social de la empresa —o como él denominó performance social— (Carroll, 1979), sino que además señaló, sin aún mencionarlo que la RSC debía ser parte de la estrategia empresarial.

Pero sobre todo, con este artículo Carroll ofreció una primera definición de la RSC en la que integró, mediante su categorización, las responsabilidades económicas (la empresa tiene como principal misión obtener beneficio de la venta de bienes y servicios producidos para la sociedad), legales (obligación de cumplir la legislación vigente), éticas (las expectativas que la sociedad posee respecto de la empresa y que van más allá del cumplimiento de la ley) y discrecionales (todas aquellas iniciativas que, no esperadas por la sociedad, parten de una iniciativa voluntaria de la empresa) (Carroll, 1979).

De alguna manera, Carroll con esta definición conjugó las ideas de Bowen, Davis, MaCguire e incluso Friedman, tratando, así, de zanjar el enfrentamiento académico suscitado años atrás.

Pero sobre todo, fue a partir de la década de los años ochenta cuando la Responsabilidad Social Corporativa comenzó a tener un sentido aplicado y práctico. Sentido que, alejándose de los orígenes de la RSC donde se buscaba alcanzar un equilibrio entre los intereses corporativos y sociales, ha estado caracterizado por el carácter instrumental que la RSC ha adquirido de cara a justificar los intereses corporativos. Particularmente con la introducción, ya en pleno siglo XXI, del concepto de valor compartido por Michael Porter y Mark Kramer.

Porter y Kramer partieron de la siguiente idea: la mayoría de los programas, contribuciones y prácticas sociales de las empresas carecen de objetivos claros en el marco de la estrategia empresarial. Esto tiene una consecuencia lógica: "los objetivos sociales y económicos son diferentes, de modo que la inversión social de una empresa va en detrimento de sus resultados económicos" (Porter \& Kramer, 2003, p. 6 e 8). 
Sin embargo, distanciándose de las tesis de Friedman, ambos autores consideraron que las empresas, cuyo funcionamiento no es ajeno ni al entorno competitivo ni a la sociedad que les rodea, pueden y deben "utilizar sus iniciativas benéficas para mejorar su contexto competitivo, es decir, la calidad del entorno empresarial en el lugar o los lugares en los que operan. Utilizar la filantropía para mejorar el contexto alinea los objetivos sociales con los económicos y mejora las perspectivas a largo plazo de una empresa" (Porter \& Kramer, 2003, p. 9).

Porter y Kramer finalmente concluyeron que "la filantropía orientada al contexto no sólo aborda el interés de una empresa, sino que beneficia a muchos a través del cambio social general" (Porter \& Kramer, 2003, p. 20).

Posteriormente en su artículo "Estrategia y sociedad", los mismos autores dan un paso más en sus tesis. Con este nuevo artículo, publicado tres años después que el anterior, asumen ya el concepto de Responsabilidad Social Corporativa.

Señalan, en el mismo, que las empresas, después de un diagnóstico previo de sus prácticas, debían incorporar la RSE en su gestión integral. Este diagnóstico debe constar, según ellos de tres etapas: $1^{\text {a }}$ ) la identificación de los impactos o consecuencias sociales que las actividades empresariales — tanto las primarias como las de apoyo- tienen, no sólo, en el entorno de la empresa, sino también, como ese entorno afecta a la competitividad empresarial; $2^{a}$ ) la gestión de la cadena de valor de tal manera que tanto los aspectos sociales como productivos queden integrados en ella, decidiendo en todo momento, de acuerdo a unas prioridades, que impactos negativos serán atenuados y cuales de los positivos serán favorecidos; $3^{\text {a }) ~ e l ~ r e c o n o c i m i e n t o ~ d e ~ c u a l e s ~ s o n ~ l a s ~ a ́ r e a s ~ d e ~ a c t u a c i o ́ n ~ p r i o r i t a r i a s ~ e n ~ e l ~ c o n t e x t o ~ c o m p e t i t i v o ~}$ en el que la empresa se desenvuelve, eligiendo tan sólo aquellas que representan una oportunidad para la creación de valor compartido (Porter \& Kramer, 2006).

La creación de valor compartido constituye la gran aportación de Porter y Kramer a la RSC. En 2011 definieron esta idea como "las políticas y las prácticas operacionales que mejoran la competitividad de una empresa a la vez que ayudan a mejorar las condiciones económicas y sociales en las comunidades donde opera” (Porter \& Kramer, 2011, p. 6).

Esta es una de las ideas que fundamentan la tesis de Antonio Madrid Pérez, quien sostiene que desde el momento en el que las empresas determinan cuales son sus 
responsabilidades y los objetivos sociales a desempeñar, eligiendo por si mismas y al margen de la legislación los mejores medios para su implementación, está desarrollando una idea de Responsabilidad Social Corporativa ajena a las obligaciones derivadas de la regulación estatal o internacional (Madrid Pérez, 2013, p. 218).

Esto, unido a la capacidad, ya mencionada, que las empresas pueden poseer de crear y producir normas de derecho blando a través, por ejemplo, de los código de conducta, es lo que Lauren Edelman ha llamado la managerización del derecho, es decir la metamorfosis que experimenta el derecho cuando es adaptado a los valores, principios y objetivos de las personas que dirigen una empresa o mánager. Se trata como la misma EDELMAN señala, de "a process by which legal ideas are refigured by managerial ways of thinking as they flow across the boundaries of legal fields and into managerial and organizational fields" (Lauren B. Edelman, Sally Riggs Fuller, \& Iona Mara-Drita, 2001, p. 1589).

Con todo, las aportaciones de Porter y Kramer han quedado consagradas en la definición que la Comisión Europea ofreció en el año 2011 de la RSC y desde la que se comprende ésta como aquel "proceso destinado a integrar las preocupaciones sociales, medioambientales y éticas, el respeto de los derechos humanos y las preocupaciones de los consumidores en sus operaciones empresariales y su estrategia básica, a fin de: 1) maximizar la creación de valor compartido para sus propietarios/accionistas y para las demás partes interesadas y la sociedad en sentido amplio; 2) identificar, prevenir y atenuar sus posibles consecuencias adversas" (Unión Europea, 2011, p. 7).

\section{Análisis y discusión}

Por tanto, estas aportaciones de Porter y Kramer que, a priori podrían parecer muy loables, no son otra cosa que la consagración de la RSC como una herramienta, no sólo llamada a dar legitimidad al orden neoliberal, sino que ha sido puesta al servicio de la competitividad, convirtiendo a la misma, tan sólo, en parte de la estrategia corporativa, que en última instancia, busca fortalecer la posición individual de la empresa en su entorno competitivo.

Por qué esta reflexión. Para responder a esta cuestión, quizás sería suficiente atender al subtítulo, como reinventar el capitalismo y liberar una oleada de innovación y crecimiento, del artículo en el que Porter y Kramer presentaron su propuesta de valor compartido. No No 
obstante, se trata de no dejar el análisis en lo meramente superficial, en el mismo artículo los autores proponen que "aprender a crear valor compartido es nuestra mejor oportunidad para volver a legitimar a las empresas" (Porter \& Kramer, 2011, p. 4). Legitimidad que, por otro lado, "permitiría el funcionamiento de la democracia a medida que los gobiernos establezcan normas que fomenten y apoyen a los negocios. La supervivencia del más apto seguirá existiendo, pero la competencia en los mercados beneficiará a la sociedad de maneras que hemos ignorado" (Porter \& Kramer, 2011, p. 18).

La RSE, de esta forma, ha tomado definitivamente, un nuevo rumbo. Más que una práctica orientada por una ética de los valores ha pasado a ser comprendida de una manera instrumental, algo capaz de generar valor para la empresa y, subsidiariamente, valor para la sociedad. Es este, precisamente, el sentido que toma cuando se observan afirmaciones como las realizadas por la Comisión Europea al señalar que "para la competitividad de las empresas es cada vez más importante un enfoque estratégico sobre RSE. Este puede reportar beneficios en cuanto a gestión de riesgos, ahorro de costes, acceso al capital, relaciones con los clientes, gestión de los recursos humanos y capacidad de innovación” (Unión Europea, 2011, p. 4).

En la práctica, esto lleva a valorar la RSE más por lo que ésta puede aportar para la empresa en términos de competitividad que por lo pueda representar para la sociedad. Al menos, esta es la conclusión a la que se puede llegar tras las palabras de José Manuel SEDES, Director del Estudio Multisectorial sobre el Estado de la Responsabilidad Corporativa de la Gran Empresa en España publicado en 2015 por el Club de Excelencia en Sostenibilidad (Club de Excelencia en Sostenibilidad, 2015).

Con todo, este conjunto de ideas hacen recordar aquellas palabras de Smith cuando en el siglo XVIII afirmaba que el agregado del interés individual representaría el progreso y el crecimiento de la sociedad (Smith, 1794).

Por tanto, frente a las tesis de Porter y Kramer, donde el interés individual —en su caso el interés de cada corporación - posee una importancia esencial, se deben contraponer ideas como las de Ostrom sobre los bienes comunes, o las de Gilligan sobre el cuidado interpersonal. Ideas en las que la centralidad se sitúa en el interés colectivo, expresado en la responsabilidad que, como sociedad, se debe asumir en relación a la protección y defensa de los bienes comunes y las personas, dando lugar, así, a un comportamiento y una ética social del bien común y del 
cuidado. Se trata, en definitiva, de asumir, en su conjunto, una nueva ética que caracterice a la responsabilidad social y que puede tener su fundamento en la ética cosmopolita de mínimos (Rueda López \& Sanchis Vidal, 2016).

\section{Conclusiones}

Más que por la razón ética de poner fin a la era del capitalismo sin escrúpulos, incapaz de alcanzar un escenario equitativo entre los derechos de la mayoría social y los intereses de la élite económica y financiera, fue la amenaza de un colapso sistémico, la falta de legitimidad social del capitalismo o el continuo cuestionamiento de su êthos y su praxis lo que llevó a que muchas voces, sin cuestionar la esencia misma del sistema neoliberal, reclamaran una revisión de las prácticas y comportamientos de sus principales agentes económicos, las empresas.

La Responsabilidad Social Corporativa fue una de las instancias señaladas para tal propósito.

Desde ese momento un conjunto de códigos éticos y de conducta, estándares, líneas directrices, principios rectores, pactos y alianzas corporativas mundiales, foros de diálogo, etc., comenzaron a ser impulsados, por los organismos rectores de la economía mundial y la comunidad internacional, con la finalidad de ofrecer a las empresas los marcos de referencia oportunos para regular su conducta.

En virtud de estos marcos, las empresas tienen la oportunidad de autorregular y autocontrolar su praxis corporativa en relación a la responsabilidad y los deberes que deben asumir en cuanto al cumplimiento de los derechos y libertades socio-laborales, el respeto de los derechos humanos y el medio ambiente, el cumplimento de las obligaciones fiscales, etc.

Se trataba, en suma, de acuerdo a la noción de gobernanza corporativa, de dotar, a su vez, a la gobernanza global del orden neoliberal de un rostro más humano.

La RSC, sostenida en lo que se ha dado en llamar el Derecho blando (soft law), se ha convertido en un fiel reflejo de las dinámicas propias del orden político-institucional neoliberal, donde el Legislador dicta, en el mejor de los casos, un conjunto de directrices mínimas, no vinculantes, destinadas a impulsar y favorecer que las empresas asuman un compromiso social, 
siendo éstas, en última instancia, quienes decidan, en función de sus estrategias e intereses comerciales, como adoptar o no las mismas.

Mientras que por un lado, en cumplimiento de las políticas neoliberales, se han recortado los servicios y prestaciones públicas, destinadas, sobre todo, a favorecer la inclusión social, la equidad y la redistribución de la riqueza, o se han debilitado las facultades legislativas de los Estados para hacer frente al poder de las empresas, por otro se ha insistido en que éstas asuman, dentro de su praxis, el mismo conjunto de acciones y políticas que los Estados estaban siendo obligados a abandonar.

Desde esta perspectiva, no sólo se asiste al desmantelamiento del Estado social y democrático de derecho, sino que, además, se fomenta que las funciones derivadas de la responsabilidad pública sean asumidas desde el ámbito corporativo, ajeno a cualquier lógica democrática.

Los derechos y libertades de las personas, antes protegidos en el marco del Estado del bienestar, ahora, en el contexto del desmantelamiento del mismo, son asumidos por las empresas en el marco de sus políticas de Responsabilidad Social Empresarial.

No sólo se han privatizado y mercantilizado servicios y prestaciones de carácter público sino que, además, la RSC puede suponer, a través del traspaso de la competencia y la responsabilidad en cuanto a la protección de los derechos y las libertades sociales del ámbito público al ámbito privado, la erosión de esta responsabilidad pública en cuanto a las garantías y la eficacia que ofrece el soft law, ámbito jurídico en el que se enmarcan las prácticas de RSC, frente al hard law, ámbito en el que reside la garantía de la protección jurídica de los derechos y libertades de la mayoría social.

\section{Bibliografía}

Annan, K. (1999). Speech of Secretary-General of the United Nations. Presentado en Annual Meeting of World Economic Forum, Davos. Speech of... Obtenido de http://goo.gl/UChOqM. Fecha de acceso: 15 de septiembre de 2013

Aras, G. 1., \& Crowther, D. (2010). A handbook of corporate governance and social responsibility. Farnham: Ashgate. 
Argandoña Ramíz, A. (2010). Más allá de la eficiencia: lecciones éticas de la crisis para la cultura empresarial, en T. Jiménez Araya (Ed.), Hacia una nueva ética económica global: innovación vs. statu quo (pp. 203-226). Barcelona: Huygens.

Bauer, R. A., \& Fenn, D. h. (1973). What is a corporate social audit? Harvard Business Review, January-February, 37-48.

Bauman, Z. (2003). Modernidad líquida. (1a ed.). Buenos Aires: Fondo de Cultura Económica.

Baxter, R. R. (1980). International Law in «Her Infinite Variety». The International and Comparative Law Quarterly, 29, 549-566.

Beck, U. (2002). La sociedad del riesgo global (2a ed.). Madrid: Siglo XXI.

Bowen, H. R. (1953). Social responsibilities of the businessman. (1st ed.). New York: Harper.

Brenner, R. (2009). La economía de la turbulencia global: las economías capitalistas avanzadas de la larga expansión al largo declive, 1945-2005. Madrid: Akal.

Bunge, M. (1980). La investigación científica: su estrategia y su filosofía. (7a ed.). Barcelona: Ariel.

Camacho Laraña, I., Fernández Fernández, J. L., \& Miralles Massanés, J. (2009). Ética de la empresa. (6a ed.). Bilbao: Desclée De Brouwer.

Carnelutti, F. (1940). Metodología del Derecho. México: Unión tipográfica editorial HispanoAmericana.

Carroll, A. B. (1979). A three-dimensional conceptual model of corporate performance. The Academy of Management Review, 4, 497-505.

Carroll, A. B. (2008). A history of corporate social responsibility: concepts and practices. In A. Crane, D. Matten, \& A. McWilliams. (Eds.). The Oxford handbook of corporate social responsibility (pp. xviii, 590 p.). New York: Oxford University Press Inc.

Castells, M. (2012). Redes de indignación y esperanza: los movimientos sociales en la era de Internet. Madrid: Alianza Editorial.

Club de Excelencia en Sostenibilidad. (2015). Estudio Multisectorial sobre el Estado de la Responsabilidad Corporativa de la Gran Empresa en España 2015. Madrid: Obtenido de https://goo.gl/Q9y4Ls. Fecha de acceso: 1 de agosto de 2018 
Committee on the financial aspects of corporate governance. (1992). The Financial aspects of corporate governance. London: Obtenido de http://goo.gl/2Lq9EA. Fecha de acceso: 23 de diciembre de 2018.

Cortina Orts, A. (2003). Las tres edades de la ética empresarial, en A. Cortina Orts. (Coord.). Construir confianza. Ética de la empresa en la sociedad de la información y las comunicaciones (226 p.). Madrid: Trotta.

Cuesta González, M. d. 1. (2004). El porqué de la responsabilidad social corporativa. Boletín económico de ICE, Información Comercial Española, 2813, pp. 45-58.

Davis, K. (1967). Understanding the Social Responsibility Puzzle: What Does the Businessman Owe to Society? Business Horizons, 10, 45-50.

Davis, K. (1973). The case for and against business assumption of social responsibilities. The Academy of Management Journal, 16, 312-322.

Davis, K., \& Blomstrom, R. L. (1966). Business and its environment. New York: McGraw-Hill.

Dempsey, A. L. (2013). Evolutions in corporate governance towards an ethical framework for business conduct. Sheffield: Greenleaf Publishing.

Edelman, L. B., Uggen, C., \& Erlanger, H. S. (1999). The Endogeneity of Legal Regulation: Grievance Procedures as Rational Myth. American Journal of Sociology, 105, 406-454.

Ferrajoli, L. (1995). Derecho y razón: teoría del garantismo penal. Madrid: Trotta.

Friedman, M. (1970). The Social Responsibility of Business is to Increase its Profits. The New York Times Magazine.

Galeano, E. (2004). Bocas del tiempo. (1a ed.). Madrid: Siglo XXI editores.

Gálvez Muñoz, L. (2000). Género, empresa e historia. Leviatán: Revista de hechos e ideas, 81, 131-168.

Gálvez Muñoz, L. (2004). Logros y retos del análisis de género en la historia económica de la empresa. Información Comercial Española, ICE: Revista de economía, 812, 77-90.

George, S. (2002). Informe Lugano (8a. ed.). Barcelona: Intermón.

Gladwin, T. N., Krause, T.-S., \& Kennelly, J. J. (1995). Beyond eco-efficiency: Towards socially sustainable business. Sustainable Development, 3, 35-43. 
Habermas, J. (1999). Problemas de legitimación en el capitalismo tardío. Madrid: Cátedra.

Klein, N. (2001). No logo: el poder de las marcas. Barcelona: Paidós Ibérica.

Laporta San Miguel, F. J. (2014). Gobernanza y soft law: nuevos perfiles jurídicos de la sociedad internacional. In A. Ruiz Miguel. (Ed.). Entre estado y cosmopolis: derecho y justicia en un mundo global. Madrid: Editorial Trotta, S.A.

Lauren B. Edelman, Sally Riggs Fuller, \& Iona Mara- Drita. (2001). Diversity Rhetoric and the Managerialization of Law. American Journal of Sociology, 106(6), 1589-1641. doi:10.1086/321303

Luelmo Millán, M. Á. (2012). La responsabilidad social corporativa en el ámbito del Derecho laboral: un instrumento económico-jurídico para un humanismo del siglo XXI. La Coruña: Netbiblo.

Madrid Pérez, A. (2013). Los deberes de las corporaciones transnacionales, en J. A. Estévez Araujo. (Ed.). El libro de los deberes las debilidades e insuficiencias de la estrategia de los derechos. Madrid: Editorial Trotta, S.A.

McGuire, J. W. (1963). Business and society. New York: McGraw-Hill.

Navarro, V. (2000). Globalización económica, poder politico y Estado del bienestar. Barcelona: Ariel.

Organización de las Naciones Unidas. (1974). Resolución sobre los efectos de las empresas transnacionales en el proceso de desarrollo y en las relaciones internacionales. Resolución E/RES/1913(LVII) (5 de diciembre de 1974), disponible en: http://goo.gl/0eRlph. Fecha de acceso: 23 de diciembre de 2018

Organización de las Naciones Unidas (2003). Resolución 58/4. Convención de las Naciones Unidas contra la Corrupción, aprobada el 31 de octubre de 2003. $n^{\circ}$ A/RES/58/4 (21 de noviembre de 2003), disponible en: https://goo.gl/phttcw. Fecha de acceso: 15 de noviembre de 2018

Organización de las Naciones Unidas. (2004). Convención de las Naciones Unidas contra la Corrupción. Viena: Oficina de las Naciones Unidas contra la Droga y el Delito. 
Organización de las Naciones Unidas. (2005). Nosotros los pueblos: la función de las Naciones Unidas en el siglo XXI. Informe del Secretario General. $n^{o}$ A/54/2000 (27 de marzo de 2000), disponible en: http://goo.gl/7HU58k. Fecha de acceso: 1 de diciembre de 2018

Porter, M. E., \& Kramer, M. R. (2003). La filantropía empresarial como ventaja competitiva. Harvard Deusto business review, 112, 6-21.

Porter, M. E., \& Kramer, M. R. (2006). Estrategia y sociedad: el vínculo entre ventaja competitiva y responsabilidad social corporativa. Harvard Business Review, 84, 42-56.

Porter, M. E., \& Kramer, M. R. (2011). La creación de valor compartido: cómo reinventar el capitalismo y liberar una oleada de innovación y crecimiento. Harvard Business Review, 89, 31-49.

Rosenau, J. N., \& Czempiel, E. O. (1992). Governance without government: order and change in world politics. Cambridge: Cambridge University Press.

Rueda López, R., \& Sanchis Vidal, A. (2016). Un modelo ético cosmopolita como propuesta para la convicancia intercultural, en Universidad de Córdoba. (Ed.). Nuevas tendencias para la interculturalidad (1a ed., 15-57). Córdoba: Universidad de Córdoba.

Sanahuja Perales, J. A. (2014). De los Objetivos del Milenio al desarrollo sostenible: Naciones Unidas y las metas globales post-2015. Anuario CEIPAZ, 49-84.

Santos, B. d. S. (2007). Más allá de la gobernanza neoliberal: el Foro Social Mundial como legalidad y política cosmopolitas subalternas, en B. d. S. Santos y C. A. Rodríguez Garavito. (Eds.). El derecho y la globalización desde abajo: hacia una legalidad cosmopolita (1a ed.). Rubí (Barcelona): Anthropos.

Satorras Fioretti, R. M. (2008). Responsabilidad social corporativa la nueva "conciencia" de las empresas y entidades. Barcelona: Bosch Editor.

Senden, L. (2004). Soft law in European Community law. Oxford: Hart.

Seoane, J., \& Taddei, E. (2001). De Seattle a Porto Alegre, pasado, presente y futuro del movimiento anti-mundialización neoliberal, en J. Seoane y E. C. Taddei. (Eds.). Resistencias mundiales: (de Seattle a Porto Alegre). (1a ed.). Buenos Aires: CLACSO. 
Smith, A. (1794). Investigación de la naturaleza y causas de la riqueza de las naciones. Tomo I. Valladolid: En la Oficina de la Viuda e Hijos de Santander.

Unión Europea (2011). Comunicación de la Comisión al Parlamento Europeo, al Consejo, al Comité Económico y Social y al Comité de las Regiones. Estrategia renovada de la UE para 2011-2014 sobre la responsabilidad social de las empresas. Comisión Europea, Comunicación COM (2011) 681 final (25 de octubre de 2011), disponible en: http://goo.gl/gXpffy. Fecha de acceso: 15 de diciembre de 2018.

Unión Europea (2013). Directiva 2013/34/UE sobre los estados financieros anuales, los estados financieros consolidados y otros informes afines de ciertos tipos de empresas, por la que se modifica la Directiva 2006/43/CE del Parlamento Europeo y del Consejo y se derogan las Directivas 78/660/CEE y 83/349/CEE del Consejo. Diario Oficial de la Unión Europea $n^{\text {o }} 56$ (29 de junio de 2013), disponible en: http://goo.gl/scmSLF. Fecha de acceso: 15 de diciembre de 2018 .

Veltmeyer, H., \& Petras, J. (1998). The dynamics of social change in Latin America. Houndmills: Macmillan Press.

Waddock, S. A. (2008). The difference makers how social and institutional entrepreneurs created the corporate responsibility movement. Sheffield, U.K.: Greenleaf Pub. Ltd. 